Bekim Osmani*, Gabriela Gerganova and Bert Müller

\title{
Biomimetic nanostructures for the silicone- biosystem interface: tuning oxygen-plasma treatments of polydimethylsiloxane
}

DOI 10.1515/ejnm-2017-0002

Received February 2, 2017; accepted March 2, 2017

Abstract: Polydimethylsiloxanes (PDMS) have drawn attention because of their applicability in medical implants, soft robotics and microfluidic devices. This article examines the formation of dedicated nanostructures on liquid submicrometer PDMS films when exposed to oxygen-plasma treatment. We show that by using a vinyl-terminated PDMS prepolymer with a molecular weight of $800 \mathrm{~g} / \mathrm{mol}$, one can bypass the need of solvent, copolymer, or catalyst to fabricate wrinkled films. The amplitude and periodicity of the wrinkles is tuned varying the thickness of the PDMS film between 150 and $600 \mathrm{~nm}$. The duration of the plasma treatment and the oxygen pressure determine the surface morphology. The amplitude was found between 30 and $300 \mathrm{~nm}$ with periodicities ranging from 500 to $2800 \mathrm{~nm}$. Atomic force microscopy was used to measure film thickness, amplitude and wrinkle periodicity. The hydrophobic recovery of the nanostructured PDMS surface, as assessed by dynamic contact angle measurements, scales with nanostructure's fineness, associated with an improved biocompatibility. The mechanical properties were extracted out of 10,000 nanoindentations on $50 \times 50-\mu \mathrm{m}^{2}$ spots. The mechanical mapping with sub-micrometer resolution reveals elastic properties according to the film morphology. Finally, we tailored the mechanical properties of a $590 \pm 120$-nm-thin silicone film to the elastic modulus of several $\mathrm{MPa}$, as required for dielectric elastomer actuators, to be used as artificial muscles for incontinence treatments.

Keywords: dielectric elastomer actuators; dynamic contact angle measurements on PDMS; local mechanical

\footnotetext{
*Corresponding author: Bekim Osmani, Department of Biomedical Engineering, Biomaterials Science Center, University of Basel, Gewerbestrasse 14, 4123 Allschwil, Switzerland,

E-mail: bekim.osmani@unibas.ch

Gabriela Gerganova and Bert Müller: Department of Biomedical Engineering, Biomaterials Science Center, University of Basel, Gewerbestrasse 14, 4123 Allschwil, Switzerland
}

properties of thin polymer films; nanometer-thin polymer films; oxygen-plasma-tuned wrinkles.

\section{Introduction}

Polydimethylsiloxane (PDMS) elastomers are inexpensive, flexible, biocompatible, optically transparent materials and their manifold fabrication techniques, such as spin coating, electrospraying, molecular beam deposition, 3D printing and molding, have led to a numerous applications (1-6). They are widely used in soft robotics, flexible electronics, microfluidic devices, medical implants, cell culture substrate and dielectric elastomer actuators, as shown in Figure 1 (3, 7-14). Its native hydrophobic surface exhibits a water contact angle of $120^{\circ} \pm 4^{\circ}$ and is mainly determined by the $\mathrm{CH}_{3}$ groups. It can easily become hydrophilic by the introduction of silanol functional groups on the PDMS surface via oxygen-plasma or ultraviolet/ozone treatments (15-18). The hydrophilicity decays with time, if stored in air, due to the migration of the smaller polymer chains from the bulk to the surface, reorientation of the polar groups and condensation of hydroxyl groups (18). The oxygen-plasma treatment forms a silica-like surface layer and its thickness increases with treatment duration and RF plasma power (19). The oxygen plasma does not only change the wettability but also alters the surface topography (20). Wavy patterns arise due to the mismatch of the thermal expansion coefficient of the formed silica-like layer and the bulk PDMS. The heating originates from the plasma itself and causes the PDMS layer to expand. Upon cooling, the bulk PDMS shrinks much more than the stiff silica-like film and exerts a compressive stress on the stiff film on top.

This stress is released by forming a wrinkling pattern on the elastomeric substrate. For thermally cross-linked PDMS films, the wrinkles can be tailored in amplitude and periodicity (21-23). It has been shown that nanostructures of plasma-treated submicrometer-thin liquid films of dissolved PDMS prepolymers with a molecular weight of $139,000 \mathrm{~g} / \mathrm{mol}$ can vary with the thickness of 


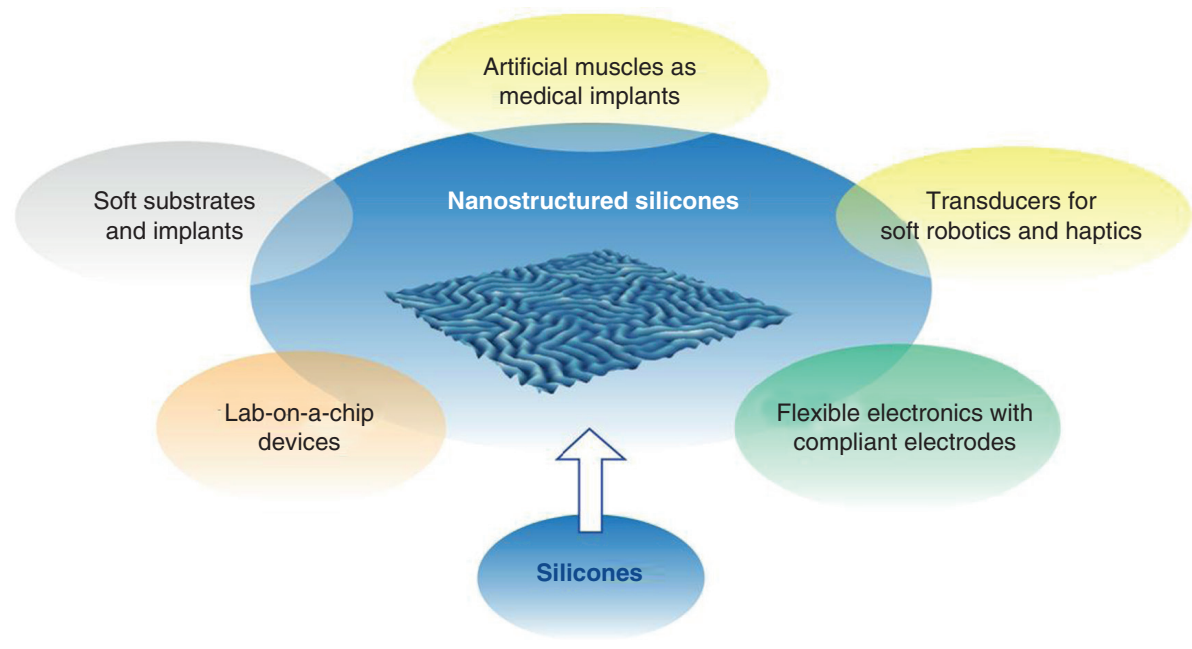

Figure 1: Applications of silicone (PDMS) elastomers: their biocompatibility, ease of use and manifold fabrication techniques have led to developments for flexible electronics, lab-on-a-chip devices, soft substrates and implants and artificial muscles as medical implants or transducers for soft robotics and haptics.

the film (24). In addition, many communications report on the estimated elastic modulus for the silica-like surface layer, which was found to be between $4 \mathrm{MPa}$ and $1.5 \mathrm{GPa}(15,19)$.

The interface between man-made material and the human body is critical for the functionality of a medical implant. In general, the body recognizes the implant as foreign body resulting in encapsulation. Several researchers have demonstrated, however, that nanostructures similar to the hydroxyapatite crystallites promote protein adsorption and can reduce the inflammatory reactions (25-29). Nanostructures on plasma-treated polymer polyetheretherketone can even support osteogenic differentiation of human mesenchymal stem cells in vitro $(30,31)$. Another key parameter that affects cell behavior is the substrate elasticity (32). Consequently, the present study on tailoring the surface morphology and elasticity tuning the plasma treatment is an essential step to realize next-generation silicone implants.

Here, we show that using a vinyl-terminated PDMS prepolymer with a molecular weight of $800 \mathrm{~g} / \mathrm{mol}$, we can bypass the need of a solvent or a cross-linking agent to fabricate PDMS films in the nanometer range. The amplitude and the periodicity of the wrinkles were tuned decreasing the film thickness, adapting the treatment duration and the oxygen pressure. Finally, we have extracted the mechanical properties of a plasma-treated PDMS film with a submicrometer resolution and showed that the average elastic modulus can be set to a few $\mathrm{MPa}$, as required for low-voltage dielectric elastomer actuators as proposed for artificial muscles (33).

\section{Materials and methods}

\section{Preparation of PDMS films}

Vinyl-terminated PDMS (V05, Gelest, Inc., Morrisville, PA, USA) with a number average molecular weight of $800 \mathrm{~g} / \mathrm{mol}$ and a kinematic viscosity of $6 \pm 2$ cSt was spun (WS-400B-6NPP/LITE/AS, Laurell Technologies Corporation, North Wales, PA, USA) onto 2-inch silicon wafers as supplied (SIEGERT WAFER GmbH, Germany). The thickness of the PDMS films was determined by scanning an edge using atomic force microscopy (AFM) in tapping mode as shown in Figure 2.

The PDMS films were prepared by pre-selected rotational speeds and spin durations to realize a variety of film thicknesses. The film thickness of $590 \pm 120 \mathrm{~nm}$ was found at a speed of $3000 \mathrm{rpm}$ applied for $2 \mathrm{~min}$. Increasing the speed to $6000 \mathrm{rpm}$ and applying the same duration, we have found a thickness of $350 \pm 50 \mathrm{~nm}$. The thickness was reduced to $150 \pm 20 \mathrm{~nm}$ using a speed of $7000 \mathrm{rpm}$ for $4 \mathrm{~min}$.

\section{Oxygen-plasma treatment of PDMS films}

The specimens were oxygen-plasma treated using a power of $200 \mathrm{~W}$ for durations of 18, 36, 72 and $144 \mathrm{~s}$ and a frequency of $40 \mathrm{kHz}$ (PICO System, Diener Electronics, Ebhausen, Germany). The oxygen partial pressure (Carbagas, Gümligen, Switzerland) was controlled varying the oxygen flow rate from 15 to $40 \mathrm{sccm}$. The application of an oxygen-plasma treatment alters the surface topography as schematically shown in Figure 3.

\section{Dynamic contact angle measurements}

The specimens were aged for a period of 180 days prior to contact angle measurements to study the hydrophobic recovery of the nanometer-thin films. They were placed on a tiltable stage of a contact angle goniometry device (OCA 15 EC, Version 2.1, DataPhysics 

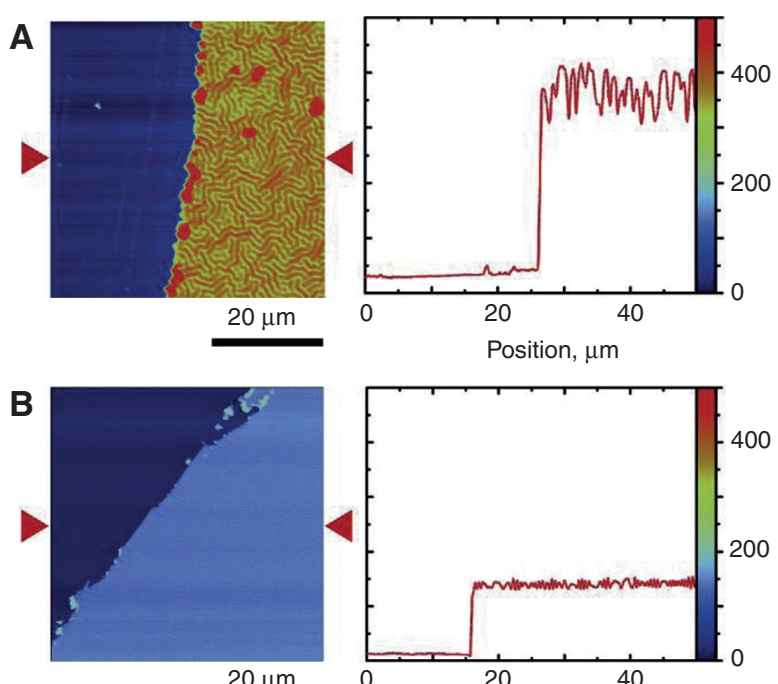

$20 \mu \mathrm{m}$

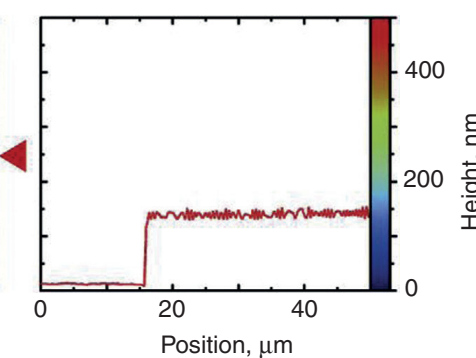

Figure 2: Determination of the PDMS film thickness: (A) AFM scan of a spin-coated and oxygen-plasma treated PDMS at a rotation speed of $6000 \mathrm{rpm}$ for a duration of $2 \mathrm{~min}$.

The profile cut according to the triangle directions on the left image shows that the wrinkling is present only on top of the film and does not affect the underlying bulk PDMS layer. (B) AFM scan and profile cut of a plasma-treated and spin-coated PDMS at a rotation speed of $7000 \mathrm{rpm}$ for a duration of $4 \mathrm{~min}$.

Instr. GmbH, Germany) supplied with LED illumination and a USB camera for video recording. Deionized water of high purity was used as the probing liquid (Elga, Purelab UHQ II, UK). A droplet of $5 \mu \mathrm{L}$ was dispensed with a glass syringe and taken up on the surface of the polymer by raising the sample stage upwards. Care was taken not to touch the needle tip with the stage.

For dynamic contact angle measurements, the tilting base unit TBU 90E (DataPhysics Instr. GmbH) was set up to perform a tilt angle of the platform from $\alpha=0^{\circ}$ to $\alpha=95^{\circ}$. The relative velocity of the rotation was set to $4.5^{\circ}$ per second with a grabbing rate of 20 frames per second. Video-based optical evaluation of the tilting was used to obtain the advancing contact angle $\theta_{a}$ and the receding contact angle $\theta_{r}$, represented in the diagrams of Figure 4. Therefore, the hysteresis $\Delta \theta=\theta_{a}-\theta_{r}$ for rolling drops, describing the difference between the advancing and receding contact angles, is calculated for $\alpha=90^{\circ}$.

The SCA 20 software (DataPhysics Instr. GmbH) was used to record and analyze all contact angle measurements. The static contact angle was recorded prior to the dynamic measurements. The apparent contact angle $\theta$ between the sessile drop and the surface of the polymer was measured by imaging the water droplet from a side view and applying a software contouring technique to calculate the angle. Droplets were visually assessed from a top view for axisymmetry before measurement.

\section{Determination of the elastic modulus $E$}

The elastic modulus of the oxygen-plasma treated PDMS films was assessed by AFM nanoindentation (NI) techniques (FlexAFM C3000, Nanosurf AG, Switzerland). For this purpose, 10,000 NIs were applied on $50 \mu \mathrm{m} \times 50 \mu \mathrm{m}$ spots using a spherical tip with a radius of $522 \pm 2$

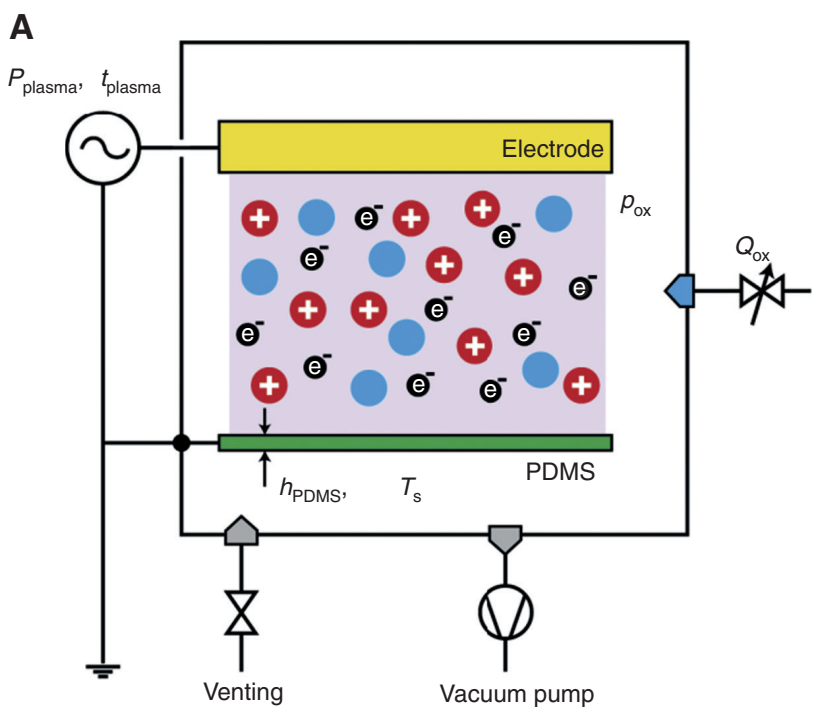

B

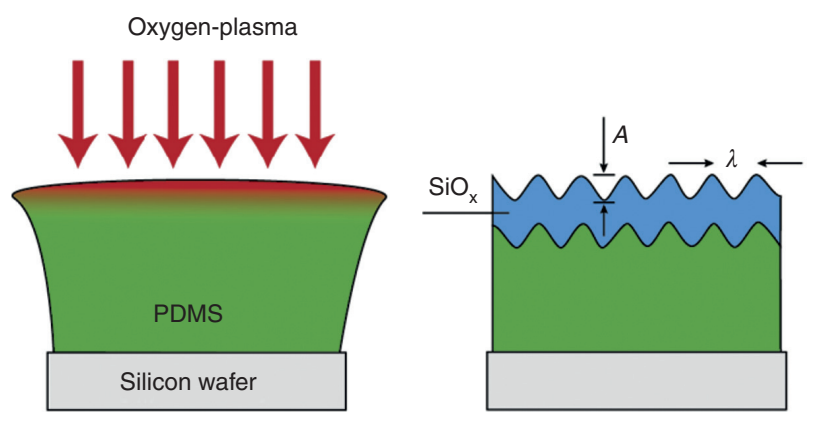

Figure 3: Formation of anisotropic and wrinkled PDMS films through oxygen plasma.

(A) Schematic of a plasma chamber showing the main parameters: power $P_{\text {plasma }}$, treatment duration $t_{\text {plasma }}$, oxygen pressure $p_{\text {ox }}$, PDMS film thickness $h_{\text {PDMS }}$ and substrate temperature $T_{\mathrm{s}}$. (B) Schematic of oxygen-plasma-treated PDMS film with the amplitude $A$ and the wavelength/periodicity $\lambda$.

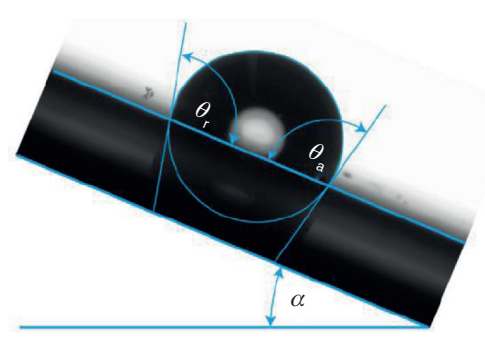

Figure 4: Schematic representation of the dynamic contact angle measurement setup, where the advancing contact angle $\theta_{\mathrm{a}}$ and the receding contact angle $\theta_{\mathrm{r}}$ are acquired for tilting angles $\alpha$ between $0^{\circ}$ and $95^{\circ}$.

nm (B500_FMR, Nanotools GmbH, Germany). The nominal spring constant of the AFM cantilever was found to be $1.9 \pm 0.1 \mathrm{~N} / \mathrm{m}$ as determined by the Sader method (34). During a single NI measurement, the sample was moved toward the AFM tip until the previously defined force of $F=200 \mathrm{nN}$ was achieved. The additional information of the $z$-piezo allowed to extract the indentation depth and calculate the mean elastic 
modulus $E$ using the Hertz contact model, which is implemented in the FLEX-ANA ${ }^{\circledR}$ software (Automated Nanomechanical Analysis, Nanosurf AG, Switzerland). Potential substrate effects were neglected since the indentation depths were less than $20 \%$ of the total film thickness.

\section{AFM imaging}

AFM images were acquired by raster-scanning a soft AFM-probe with a radius smaller than $10 \mathrm{~nm}$ (Tap190Al-G probe, NanoAndMore GmbH, Wetzlar, Germany) in tapping mode (vibration amplitude $2.0 \mathrm{~V}$, set point $60 \%$ ) using a FlexAFM System (Nanosurf AG, Liestal, Switzerland). A total of 512 lines at a speed of $1 \mathrm{~s}$ per line were acquired for each image. The raw data were leveled by subtracting a mean plane and removing a polynomial background of the first degree. Root-mean-square (RMS) roughness values were calculated by the open source software Gwyddion, Version 2.41 .

\section{Results}

AFM scans and dynamic contact angle measurements of the oxygen-plasma treated PDMS films $\left(P_{\text {plasma }}=200 \mathrm{~W}\right.$, $t_{\text {plasma }}=60 \mathrm{~s}, p_{\text {ox }}=45 \mathrm{~Pa}, T_{\mathrm{s}}=25^{\circ} \mathrm{C}$ ) are shown in Figure 5. The amplitude $A$, as extracted out the height distribution diagram and the periodicity/wavelength $\lambda$ of the wrinkles are summarized in Table 1 . The periodicity $\lambda$ is calculated using 2D fast Fourier transform (2D FFT) of the related AFM image. The periodicity $\lambda$ and the amplitude $A$ decrease with film thickness.

We show in Figure 6 dynamic contact angle measurements and AFM images of surfaces of $350 \pm 50 \mathrm{~nm}$ PDMS films, which were oxygen-plasma treated $\left(P_{\text {plasma }}=200 \mathrm{~W}\right.$, $t_{\text {plasma }}=60 \mathrm{~s}, T_{\mathrm{s}}=25^{\circ} \mathrm{C}$ ) at oxygen pressures from $p_{\text {ox }}=18$ to $p_{\text {ox }}=45 \mathrm{~Pa}$. The amplitude $A$, as extracted out the height distribution diagram and the periodicity/wavelength $\lambda$ of the wrinkles are summarized in Table 2 . It is found that the periodicity $\lambda$ and the amplitude $A$ increase at lower oxygen pressures $p_{\text {ox }}$.

PDMS films with a thickness of $350 \pm 50 \mathrm{~nm}$ were oxygen-plasma treated $\left(P_{\text {plasma }}=200 \mathrm{~W}, p_{\text {ox }}=45 \mathrm{~Pa}, T_{\mathrm{s}}=25^{\circ} \mathrm{C}\right)$ by varying the treatment duration from 18 to $144 \mathrm{~s}$ are shown in Figure 7. The amplitude $A$, as extracted out the height
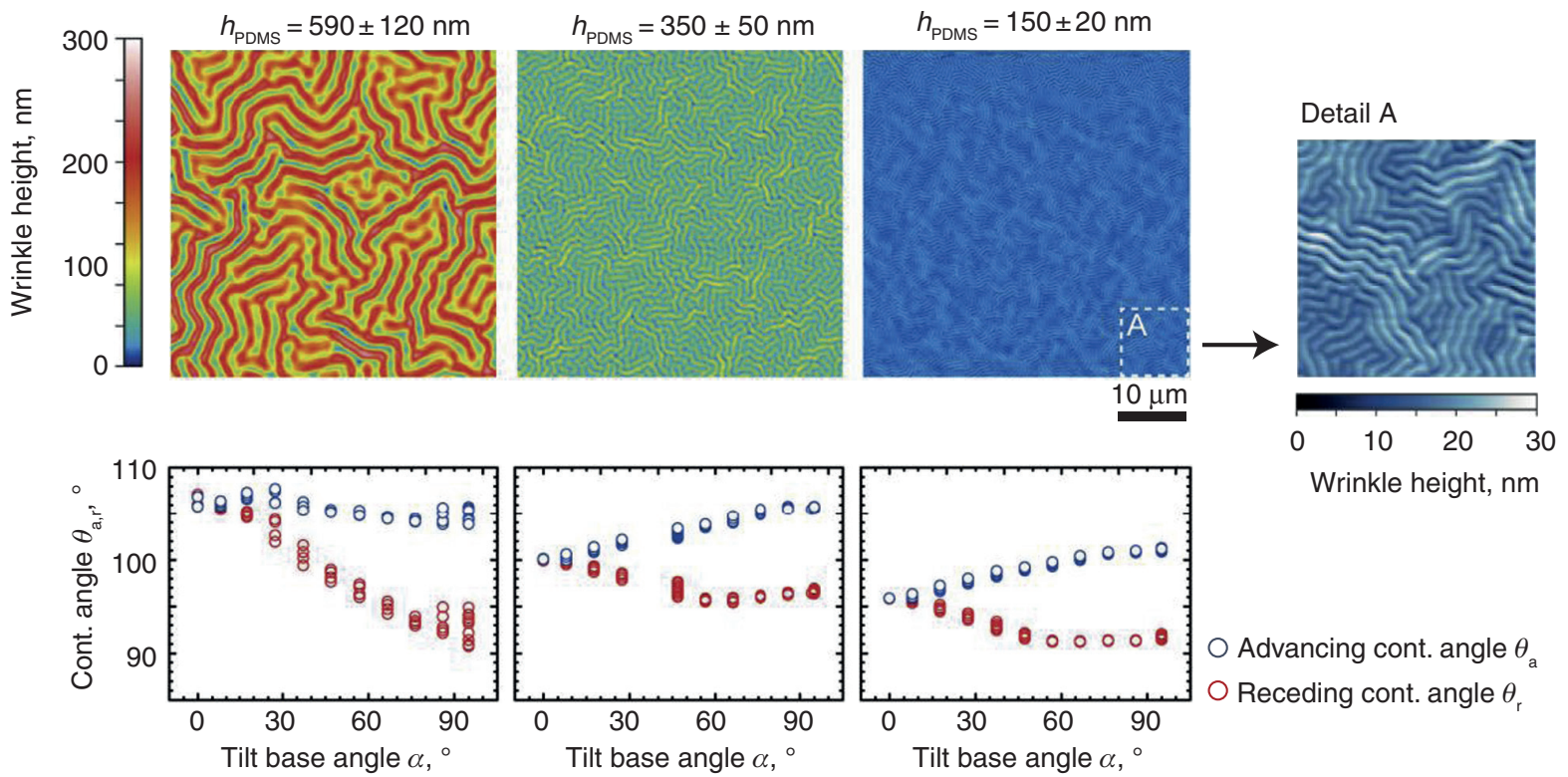

Advancing cont. angle $\theta_{\text {a }}$ Receding cont. angle $\theta_{r}$

Figure 5: AFM scans and dynamic contact angle measurements of PDMS films with varying film thicknesses $h_{\text {PDMs }}$. The amplitude $A$ and periodicity $\lambda$ of the wrinkles show a positive correlation with the film thickness. The contact angle $\theta$ decreased with decrement in film thickness from $107^{\circ} \pm 1^{\circ}$ to $96^{\circ} \pm 1^{\circ}$ and the hysteresis $\Delta \theta$ shows a tendency towards smaller values.

Table 1: Contact angle, periodicity and amplitude of plasma-treated PDMS films $\left(P_{\text {plasma }}=200 \mathrm{~W}, t_{\text {plasma }}=60 \mathrm{~s}, p_{\text {ox }}=45 \mathrm{~Pa}, T_{\mathrm{s}}=25^{\circ} \mathrm{C}\right)$ for selected film thicknesses.

\begin{tabular}{lrrrrr}
\hline $\begin{array}{l}\text { Film thickness } \\
\boldsymbol{h}_{\text {PDMS }}, \mathbf{n m}\end{array}$ & $\begin{array}{r}\text { Contact } \\
\text { angle } \boldsymbol{\theta}, \text { deg }\end{array}$ & $\begin{array}{r}\text { Hysteresis } \boldsymbol{\Delta} \boldsymbol{\theta} \\
\text { at } \boldsymbol{\alpha}=\mathbf{9 0}^{\circ}, \mathbf{d e g}\end{array}$ & $\begin{array}{r}\text { Periodicity of the } \\
\text { wrinkles } \boldsymbol{\lambda}, \mathrm{nm}\end{array}$ & $\begin{array}{r}\text { Amplitude } \\
\boldsymbol{A}, \mathbf{n m}\end{array}$ & $\begin{array}{r}\text { Ratio amplitude to } \\
\text { film thickness } \boldsymbol{A} / \boldsymbol{h}_{\text {PDMs }}\end{array}$ \\
\hline $590 \pm 120$ & $107 \pm 1$ & $11 \pm 1$ & $2800 \pm 200$ & $\approx 200$ & $\approx 1 / 3$ \\
$350 \pm 50$ & $99 \pm 1$ & $10 \pm 1$ & $1100 \pm 100$ & $\approx 90$ & $\approx 1 / 4$ \\
$150 \pm 20$ & $96 \pm 1$ & $9 \pm 1$ & $500 \pm 100$ & $\approx 30$ & $\approx 1 / 5$ \\
\hline
\end{tabular}




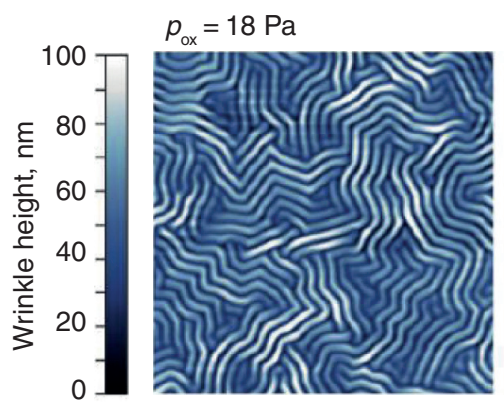

O Advancing cont. angle $\theta_{\mathrm{a}}$

$\bigcirc$ Receding cont. angle $\theta_{\mathrm{r}}$

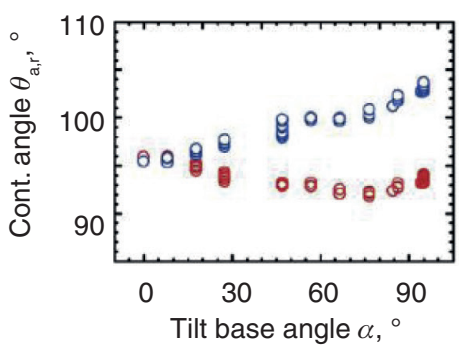

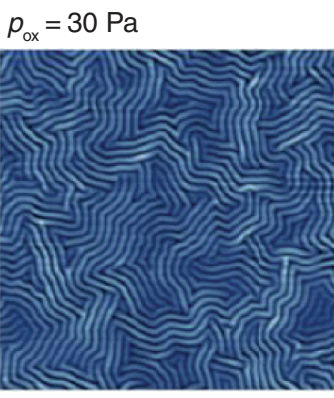

$p_{\text {ox }}=35 \mathrm{~Pa}$

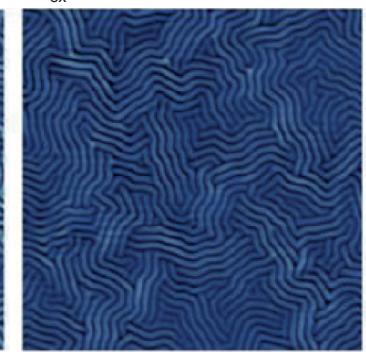

$p_{\text {ox }}=45 \mathrm{~Pa}$

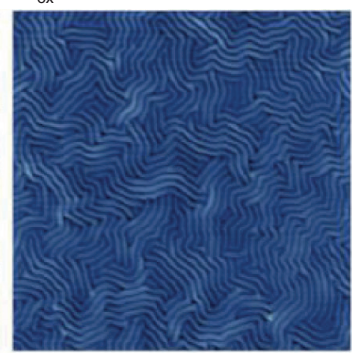

$15 \mu \mathrm{m}$
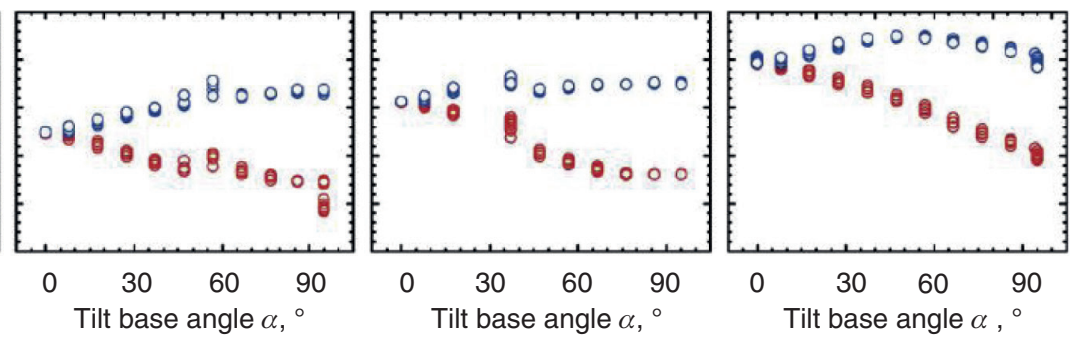

Figure 6: AFM images and dynamic contact angle measurements of $350 \pm 50 \mathrm{~nm}$ PDMS films at oxygen pressures $p_{\text {ox }}$ indicated. The amplitude $A$ and periodicity $\lambda$ of the wrinkles reversely relate with the oxygen pressure $p_{\text {ox }}$. The initial contact angle $\theta$ increased slightly with increment in oxygen pressure $p_{\text {ox }}$ from $96^{\circ} \pm 1^{\circ}$ to $105^{\circ} \pm 1^{\circ}$ and the hysteresis $\Delta \theta$ at $\alpha=90^{\circ}$ remained constant at $10^{\circ} \pm 1^{\circ}$.

distribution diagram, and the periodicity/wavelength $\lambda$ of the wrinkles are listed in Table 3 . The periodicity $\lambda$ was calculated using 2D fast Fourier transform (2D FFT) of the related AFM images and are found to remain constant at $1100 \pm 100 \mathrm{~nm}$ for the selected conditions.

A PDMS film with a thickness of $590 \pm 120 \mathrm{~nm}$ was plasma-treated at an RF power $P_{\text {plasma }}=200 \mathrm{~W}$ for a total duration of $t_{\text {plasma }}=60 \mathrm{~s}$. The mechanical mapping with submicrometer resolution, shown in Figure 8, reveals topology-dependent elastic properties. It exhibits elastic moduli of $1.8 \pm 0.6$ and $3.9 \pm 1.7 \mathrm{MPa}$, which are in the range of interest for dielectric elastomer actuators. Substrate effects can be neglected because the PDMS film was $590 \pm 120 \mathrm{~nm}$ thick, which is large compared to the NI depth of $150 \pm 50 \mathrm{~nm}$. We can also exclude geometrical artifacts because the spherical tip has a radius of $522 \pm 2$ $\mathrm{nm}$, which is small with respect to the periodicity of the wrinkles corresponding to $2800 \pm 200 \mathrm{~nm}$. In addition, the map reveals that the material on the hills is significantly stiffer with respect to the one located in the valleys.

\section{Discussion and conclusions}

Our results show that using a vinyl-terminated PDMS prepolymer with a number average molecular weight of $800 \mathrm{~g} / \mathrm{mol}$, one can easily bypass the use of any solvent or curing agent to fabricate nanostructured PDMS elastomer films as thin as hundreds of nanometer.

As plasma treatments do not only change the surface morphology but also influence the chemistry and the related mechanics of the surface layers, the cross-linking

Table 2: Changing the oxygen pressure $p_{\text {ox }}$ and keeping all the other parameters constant for plasma-treated $350 \pm 50 \mathrm{~nm}$ PDMS films $\left[P_{\text {plasma }}=200 \mathrm{~W}, t_{\text {plasma }}=60 \mathrm{~s}, h_{\text {PDMS }}=350 \pm 50 \mathrm{~nm}, T_{\mathrm{s}}=25^{\circ} \mathrm{C}\right]$.

\begin{tabular}{|c|c|c|c|c|}
\hline $\begin{array}{l}\text { Oxygen pressure } \\
p_{\text {ox }}, \mathrm{Pa}\end{array}$ & $\begin{array}{r}\text { Contact } \\
\text { angle } \boldsymbol{\theta}, \text { deg }\end{array}$ & $\begin{array}{r}\text { Hysteresis } \Delta \theta \\
\text { at } \alpha=90^{\circ}, \text { deg }\end{array}$ & $\begin{array}{r}\text { Periodicity of the } \\
\text { wrinkles } \lambda, \mathrm{nm}\end{array}$ & $\begin{array}{r}\text { Amplitude } \\
A, \mathrm{~nm}\end{array}$ \\
\hline 18 & $96 \pm 1$ & $10 \pm 1$ & $1800 \pm 200$ & $\approx 80$ \\
\hline 30 & $98 \pm 1$ & $10 \pm 1$ & $1400 \pm 150$ & $\approx 70$ \\
\hline 35 & $96 \pm 1$ & $10 \pm 1$ & $1300 \pm 150$ & $\approx 30$ \\
\hline 45 & $105 \pm 1$ & $10 \pm 1$ & $1100 \pm 100$ & $\approx 25$ \\
\hline
\end{tabular}



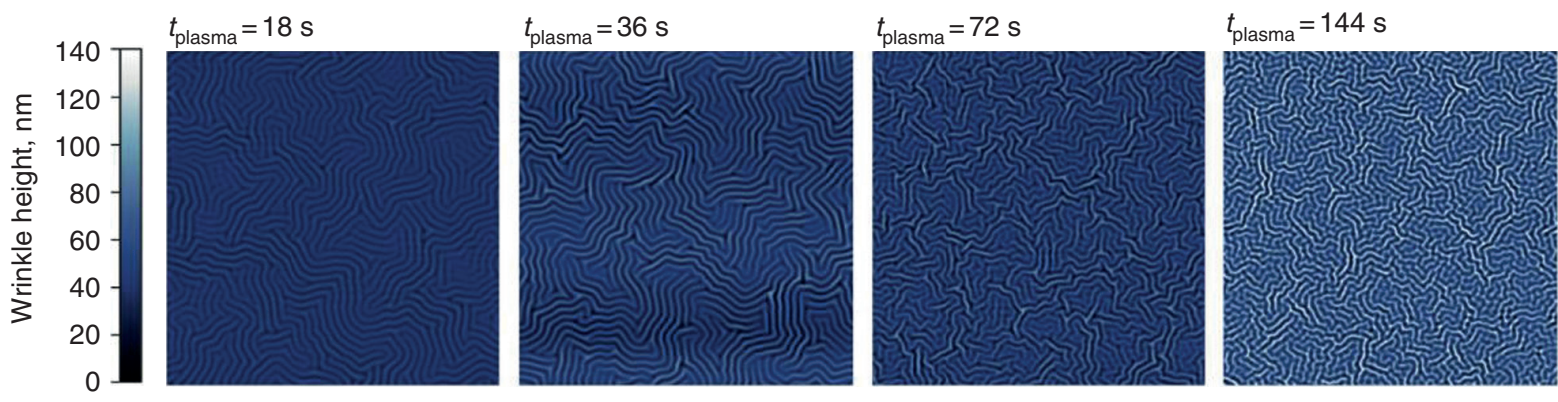

Advancing cont. angle $\theta_{\mathrm{a}}$

Receding cont. angle $\theta_{\mathrm{r}}$
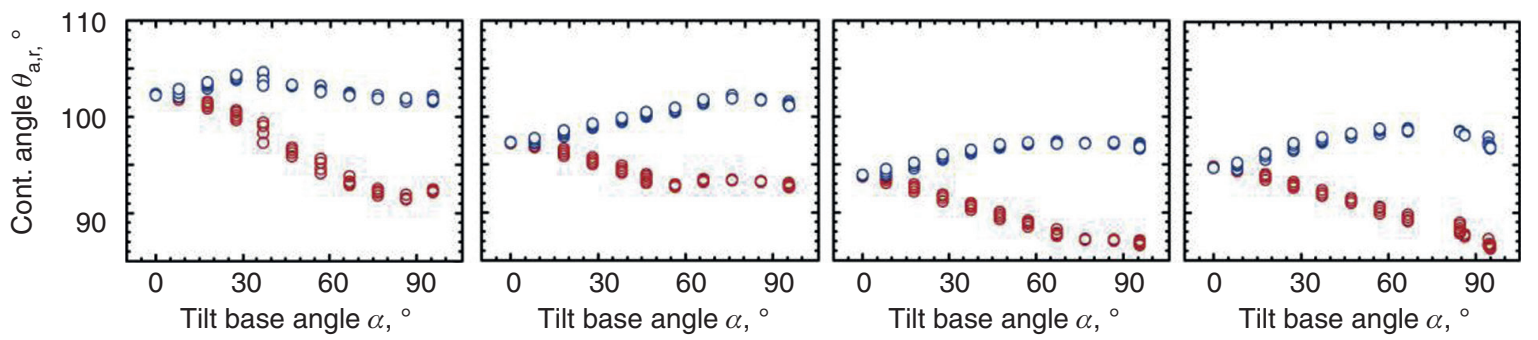

Figure 7: AFM scans and dynamic contact angle measurements of $350 \pm 50 \mathrm{~nm}$ PDMS films, plasma treated for different durations $t_{\text {plasma }}$. The amplitude $A$ increased 10 -fold up to $100 \mathrm{~nm}$ for $t_{\text {plasma }}=144 \mathrm{~s}$, whereas the periodicity $\lambda$ remained constant at $1100 \pm 100 \mathrm{~nm}$. The contact angle $\theta$ decreased from $102^{\circ} \pm 1^{\circ}$ to $95^{\circ} \pm 1^{\circ}$ and the hysteresis $\Delta \theta$ at $\alpha=90^{\circ}$ remained constant at $10^{\circ} \pm 1^{\circ}$.

Table 3: Increasing the treatment duration leads to larger amplitudes.

\begin{tabular}{|c|c|c|c|c|}
\hline $\begin{array}{l}\text { Treatment } \\
\text { duration } t_{\text {plasma }}, \mathbf{s} \\
\end{array}$ & $\begin{array}{r}\text { Contact } \\
\text { angle } \theta, \text { deg }\end{array}$ & $\begin{array}{r}\text { Hysteresis } \Delta \theta \\
\text { at } \alpha=90^{\circ}, \operatorname{deg} \\
\end{array}$ & $\begin{array}{r}\text { Periodicity of the } \\
\text { wrinkles } \lambda, \mathrm{nm}\end{array}$ & $\begin{array}{r}\text { Amplitude } \\
A, \mathrm{~nm}\end{array}$ \\
\hline 18 & $102 \pm 1$ & $10 \pm 1$ & $1100 \pm 100$ & $\approx 10$ \\
\hline 36 & $97 \pm 1$ & $9 \pm 1$ & $1100 \pm 100$ & $\approx 40$ \\
\hline 72 & $94 \pm 1$ & $10 \pm 1$ & $1100 \pm 100$ & $\approx 60$ \\
\hline 144 & $95 \pm 1$ & $10 \pm 1$ & $1100 \pm 100$ & $\approx 100$ \\
\hline
\end{tabular}

All other parameters were kept constant for the plasma-treated PDMS films $\left[P_{\text {plasma }}=200 \mathrm{~W}, p_{\text {ox }}=45 \mathrm{~Pa}, h_{\mathrm{PDMS}}=350 \pm 50 \mathrm{~nm}, T_{\mathrm{s}}=25^{\circ} \mathrm{C}\right]$.

mechanism via reactive species generated through photoionization and ion bombardments have to be considered. As previously shown, the irradiation of PDMS with light at wavelengths of approximately $170 \mathrm{~nm}$ leads to scissions of the $\mathrm{Si}-\mathrm{CH}_{3}$ and $\mathrm{Si}-\mathrm{CH}_{2}-\mathrm{H}$ bonds. The resulting unsaturated bonds give rise to a three-dimensional elastomer network $(33,35)$. It should be noted that we observed a strong adherence of the PDMS films to the Si wafer. It cannot be removed simply by wiping or by washing out in ethyl acetate. This effect could be associated with the comparably low wrinkle periodicity of the thinner films because the plasma treatment also affects layers well below the surface.

The wrinkling phenomena of a simple bilayer system are understood (36). It has been shown that a compressed film on a much less stiff elastomer undergoes wrinkling to relieve the intrinsic compressive stress. The orientation of the wrinkles is generally random. Buckling models can precisely predict the periodicity of these randomly oriented wrinkles $\lambda(21)$ :

$$
\lambda=2 \pi h_{\mathrm{f}}\left(\frac{\left(1-v_{\mathrm{f}}^{2}\right) E_{\mathrm{f}}}{3\left(1-v_{\mathrm{s}}^{2}\right) E_{\mathrm{s}}}\right)^{1 / 3},
$$

where $h_{\mathrm{f}}$ corresponds to the thickness of the stiffer film, $E_{\mathrm{s}, \mathrm{f}}$ and $v_{\mathrm{s}, \mathrm{f}}$ are the elastic moduli and the Poisson's ratios of the two components, respectively. This two-layer model, however, does only partially account for the plasma-treated PDMS. The plasma treatment does not only produce a nanometer-thin film of increasing thickness but probably also changes the elastic properties of the underlying elastomer. Assuming that unchanged plasma treatment conditions result in alike parameters 

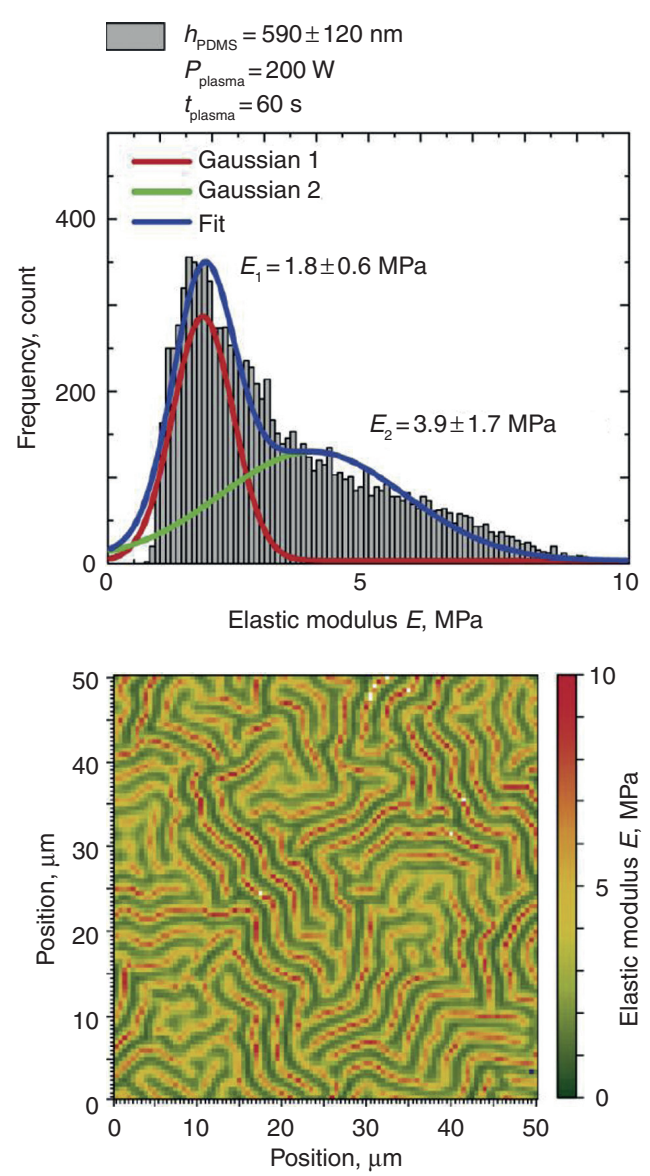

Figure 8: Stiffness histogram and map out of 10,000 NIs on a $50 \times 50 \mu \mathrm{m}^{2}$ spot.

The submicrometer mechanical mapping of the spot reveals softer valleys with an elastic modulus of $E_{1}=1.8 \pm 0.6 \mathrm{MPa}$ compared to hills showing an elastic modulus of $E_{2}=3.9 \pm 1.7$ $\mathrm{MPa}$. Substrate effects can be neglected since the NIs depth were below $200 \mathrm{~nm}$.

$h_{\mathrm{f}}$ and $E_{\mathrm{f}}$ and the thinner films are more intensely plasmatreated resulting in an increased $E_{s}$, according to Equation (1), the ratio $E_{\mathrm{f}} / E_{\mathrm{s}}$ becomes smaller, giving rise to a shorter periodicity. We have observed such a behavior, as listed in Table 1 and represented in Figure 5. The periodicity $\lambda$ decreases with the decrement in film thickness. The amplitude $A$ of the wrinkling pattern is proportional to the square root of the buckling strain $\varepsilon$ (36). Neglecting the impact of the Poisson coefficients, one finds

$$
\varepsilon=\left(\frac{9 E_{s}^{2}}{64 E_{f}^{2}}\right)^{1 / 3} .
$$

As the treatment duration $t_{\text {plasma }}$ could influence the bulk elastic modulus $E_{\mathrm{s}}$, the model correlates well with our findings. For $t_{\text {plasma }}=144 \mathrm{~s}$, we have demonstrated a 10 -fold amplitude increase. According to Equation (1), one should observe a periodicity decrease. Instead, the experiments show a constant value. Here, we speculate that the thickness $h_{\mathrm{f}}$ is increasing with treatment duration. Reducing the oxygen pressure $p_{\text {ox }}$, as given by the data in Table 2 and Figure 6, one can reasonably assume that $E_{\mathrm{s}}$ increases. Therefore, both the amplitude $A$ and the periodicity $\lambda$ are similarly influenced, as described by means of Equations (1) and (2).

The mechanical properties of a plasma-treated PDMS film locally measured with submicrometer precision reveals that the elastic modulus of the material in valleys differs from that on hills. The difference by a factor of two is surprisingly high. So far, one can only speculate on the reasons. We know the cross-sectional profiles of wrinkle's networks. Geometrical phenomena related to the sinusoidal microstructure could be a reason but probably do not explain such a large effect. We further know that the wrinkle formation is associated with significant material transport (37). Therefore, one can reasonably assume a thickness modulation of the silicate layer according to the wrinkle structure.

The wetting of the aged, nanostructured PDMS films, as studied by contact angle goniometry, was improved for the ones with smaller amplitude and wrinkle periodicity.

Smaller contact angles are also found on samples treated at lower oxygen pressures. This could be due to the higher $\mathrm{SiO}_{\mathrm{x}}$ concentration on the surface exposed to increased number of ion bombardments at lower oxygen pressures $p_{\text {ox }}$. The hysteresis $\Delta \theta$ at $\alpha=90^{\circ}$ is found to be constant for all samples showing a value of $10^{\circ} \pm 1^{\circ}$. All samples were stored under ambient conditions for a period of 180 days. The contact angle of water on a surface plasma-treated with $P_{\text {plasma }}=200 \mathrm{~W}, t_{\text {plasma }}=18 \mathrm{~s}$, $T_{\mathrm{s}}=20^{\circ} \mathrm{C}$ and $p_{\text {ox }}=45 \mathrm{~Pa}$ corresponded $49^{\circ} \pm 1^{\circ}$ several hours after fabrication and increased to $77^{\circ} \pm 1^{\circ}$ and $85^{\circ} \pm 1^{\circ}$ after 2 and 3 weeks, respectively. It is worth mentioning that plasma-polymerized PDMS films recover similarly to plasma-treated cross-linked PDMS films. Further investigations are required to analyze the prospects and challenges of these wrinkled elastomer films to serve as a support for stretchable electrodes and their integration in the field of artificial muscles and medical implants.

Acknowledgments: The financial support of the nanotera.ch initiative (grant/award no. "SmartSphincter RTD2013”), project SmartSphincter, as well as the Swiss Nanoscience Institute (SNI) for the financial contribution to the AFM is gratefully acknowledged. The authors also 
thank Dr. Thomas Pfohl and Dr. Tino Töpper for the discussion of the results presented.

Conflict of interest statement: Authors state no conflict of interest. All authors have read the journal's publication ethics and publication malpractice statement available at the journal's website and hereby confirm that they comply with all its parts applicable to the present scientific work.

\section{References}

1. Poulin A, Rosset S, Shea HR. Printing low-voltage dielectric elastomer actuators. Appl Phys Lett 2015;107:244104.

2. Hinton TJ, Hudson A, Pusch K, Lee A, Feinberg AW. 3D printing PDMS elastomer in a hydrophilic support bath via freeform reversible embedding. ACS Biomater Sci Eng 2016;2:1781-6.

3. Rosset S, Shea HR. Small, fast, and tough: Shrinking down integrated elastomer transducers. Appl Phys Rev 2016;3:031105.

4. Weiss FM, Madsen FB, Töpper T, Osmani B, Leung V, Müller B. Molecular beam deposition of high-permittivity polydimethylsiloxane for nanometer-thin elastomer films in dielectric actuators. Mater Des 2016;105:106-13.

5. Weiss FM, Töpper T, Osmani B, Deyhle H, Kovacs G, Müller B. Thin film formation and morphology of electrosprayed polydimethylsiloxane. Langmuir 2016;32:3276-83.

6. Weiss FM, Töpper T, Osmani B, Peters S, Kovacs G, Müller B. Electrospraying nanometer-thin elastomer films for low-voltage dielectric actuators. Adv Electron Mater 2016;2:1500476.

7. Sia SK, Whitesides GM. Microfluidic devices fabricated in poly(dimethylsiloxane) for biological studies. Electrophoresis 2003;24:3563-76.

8. Lee JN, Jiang X, Ryan D, Whitesides GM. Compatibility of mammalian cells on surfaces of poly(dimethylsiloxane). Langmuir 2004;20:11684-91.

9. O'Halloran A, O'Malley F, McHugh P. A review on dielectric elastomer actuators, technology, applications, and challenges. J Appl Phys 2008;104:071101.

10. Brochu P, Pei Q. Advances in dielectric elastomers for actuators and artificial muscles. Macromol Rapid Commun 2010;31:10-36.

11. Shepherd RF, Ilievski F, Choi W, Morin SA, Stokes AA, Mazzeo $A D$, et al. Multigait soft robot. Proc Natl Acad Sci USA 2011;108:20400-3.

12. Anderson IA, Gisby TA, McKay TG, O’Brien BM, Calius EP. Multifunctional dielectric elastomer artificial muscles for soft and smart machines. J Appl Phys 2012;112:041101.

13. Osmani B, Töpper T, Deschenaux C, Nohava J, Weiss FM, Leung $\mathrm{V}$, et al. Micro- and nanostructured electro-active polymer actuators as smart muscles for incontinence treatment. AIP Conf Proc 2015;1646:91-100.

14. Fattorini E, Brusa T, Gingert C, Hieber S, Leung V, Osmani B, et al. Artificial muscle devices: innovations and prospects for fecal incontinence treatment. Ann Biomed Eng 2016;44:1-15.

15. Hillborg H, Ankner JF, Gedde UW, Smith GD, Yasuda HK, Wikström K. Crosslinked polydimethylsiloxane exposed to oxygen plasma studied by neutron reflectometry and other surface specific techniques. Polymer 2000;41:6851-63.
16. Efimenko K, Wallace WE, Genzer J. Surface modification of sylgard-184 poly(dimethyl siloxane) networks by ultraviolet and ultraviolet/ozone treatment. J Colloid Interface Sci 2002;254:306-15.

17. Lawton A, Price CR, Runge AF, Doherty WJ, Saavedra SS. Air plasma treatment of submicron thick PDMS polymer films: effect of oxidation time and storage conditions. Colloids Surf A 2005;253:213-5

18. Bodas D, Khan-Malek C. Hydrophilization and hydrophobic recovery of PDMS by oxygen plasma and chemical treatment an SEM investigation. Sens Actuators B 2007;123:368-73.

19. Béfahy S, Lipnik P, Pardoen T, Nascimento C, Patris B, Bertrand $P$, et al. Thickness and elastic modulus of plasma treated PDMS silica-like surface layer. Langmuir 2010;26:3372-5.

20. Bowden N, Brittain S, Evans AG, Hutchinson JW, Whitesides GM. Spontaneous formation of ordered structures in thin films of metals supported on an elastomeric polymer. Nature 1998;393:146-9.

21. Bowden N, Huck WT, Paul KE, Whitesides GM. The controlled formation of ordered, sinusoidal structures by plasma oxidation of an elastomeric polymer. Appl Phys Lett 1999;75:2557-9.

22. Chua DB, Ng HT, Li SF. Spontaneous formation of complex and ordered structures on oxygen-plasma-treated elastomeric polydimethylsiloxane. Appl Phys Lett 2000;76:721-3.

23. Tserepi A, Gogolides E, Tsougeni K, Constantoudis V, Valamontes ES. Tailoring the surface topography and wetting properties of oxygen-plasma treated polydimethylsiloxane. J Appl Phys 2005;98:113502.

24. Evensen HT, Jiang H, Gotrik KW, Denes F, Carpick RW. Transformations in wrinkle patterns: cooperation between nanoscale cross-linked surface layers and the submicrometer bulk in wafer-spun, plasma-treated polydimethylsiloxane. Nano Lett 2009;9:2884-90.

25. Müller B. Natural formation of nanostructures: from fundamentals in metal heteroepitaxy to applications in optics and biomaterials science. Surf Rev Lett 2001;08:169-228.

26. Müller B, Riedel M, Michel R, De Paul SM, Hofer R, Heger D, et al. Impact of nanometer-scale roughness on contact-angle hysteresis and globulin adsorption. J Vac Sci Technol B 2001;19:1715-20.

27. Riedel M, Müller B, Wintermantel E. Protein adsorption and monocyte activation on germanium nanopyramids. Biomaterials 2001;22:2307-16.

28. Dalby MJ, Gadegaard N, Tare R, Andar A, Riehle MO, Herzyk $P$, et al. The control of human mesenchymal cell differentiation using nanoscale symmetry and disorder. Nat Mater 2007;6:997-1003.

29. Kolind K, Leong KW, Besenbacher F, Foss M. Guidance of stem cell fate on 2D patterned surfaces. Biomaterials 2012;33:6626-33.

30. Waser-Althaus J, Padeste C, Köser J, Pieles U, Peters K, Müller B. Nanostructuring polyetheretherketone for medical implants. Eur J Nanomed 2012;4:7-15.

31. Waser-Althaus J, Salamon A, Waser M, Padeste C, Kreutzer M, Pieles $U$, et al. Differentiation of human mesenchymal stem cells on plasma-treated polyetheretherketone. J Mater Sci Mater Med 2014;25:515-25.

32. Engler AJ, Sen S, Sweeney HL, Discher DE. Matrix elasticity directs stem cell lineage specification. Cell 2006;126:677-89.

33. Töpper T, Weiss FM, Osmani B, Bippes C, Leung V, Müller B. Siloxane-based thin films for biomimetic low-voltage dielectric actuators. Sens Actuators A 2015;233:32-41. 
34. John ES, James WM, Paul M. Calibration of rectangular atomic force microscope cantilevers. Rev Sci Instrum 1999;70:3967-9.

35. Vasilets VN, Kovalchuk AV, Ponomarev AN. Photooxidation of siloxane polymers under vacuum ultraviolet irradiation. J Photopolym Sci Technol 1994;7:165-74.

36. Schweikart A, Fery A. Controlled wrinkling as a novel method for the fabrication of patterned surfaces. Microchim Acta 2009;165:249-63.

37. Müller B, Deyhle H, Mushkolaj S, Wieland M. The challenges in artificial muscle research to treat incontinence. Swiss Med. Wkly 2009;139:591-5.

\section{Bionotes}

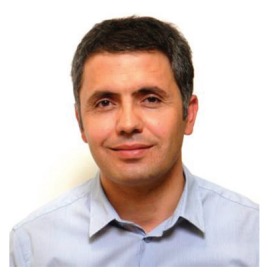

\author{
Bekim Osmani \\ Department of Biomedical Engineering, \\ Biomaterials Science Center, University of \\ Basel, Gewerbestrasse 14, 4123 Allschwil, \\ Switzerland \\ bekim.osmani@unibas.ch
}

Bekim Osmani is a PhD student at the Biomaterials Science Center at the University of Basel. He did his BSc in Mechanical Engineering and his MSc in Biomedical Engineering and Robotics at the Swiss Federal Institute of Technology in Zurich (ETHZ) in 2002. After several years of experience in academia and industry, he is currently working toward his $\mathrm{PhD}$ degree in nanosciences. His research interests include molecular beam deposition and electrospraying of nanometer thin elastomer films, atomic force microscopy, nanoindentation techniques and mechanical properties of nanometer thin film, polymeric implants and biomedical applications for low-voltage dielectric elastomer actuators.

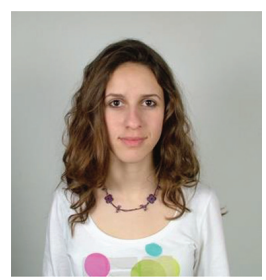

\section{Gabriela Gerganova}

Department of Biomedical Engineering, Biomaterials Science Center, University of Basel, Gewerbestrasse 14, 4123 Allschwil, Switzerland

Gabriela Gerganova, 22 years old, is an integrated master's student of pharmacology at University of Glasgow. She performs her placement year at the Biomaterials Science Centre under the direction of Prof. Bert Müller. Her research focus is on the formulation, characterization and safety of liposome-based nanocontainers for targeted drug delivery. During her internship, she has expanded her practical skills in biomedical engineering with the help of on-site laboratory facilities.

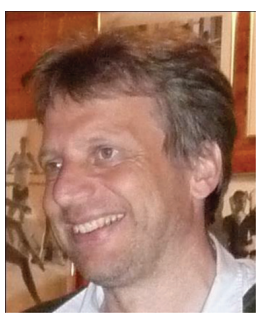

Bert Müller

Department of Biomedical Engineering, Biomaterials Science Center, University of Basel, Gewerbestrasse 14, 4123 Allschwil, Switzerland

Bert Müller received a diploma in mechanical engineering, Berlin 1982, followed by MSc degrees in Physics and English both from the Dresden University of Technology in 1989. In 1994, he obtained a PhD in experimental physics from the University of Hannover, Germany. For his achievements, he was granted with the Morton M. Traum Award of the American Vacuum Society in 1994. From 1994 to 2001, he worked as a researcher at the Paderborn University, Germany, as Feodor Lynen Fellow and research associate at the EPF Lausanne, Switzerland and as team leader at the Physics Department, Materials Department and Department of Information Technology and Electrical Engineering at ETH Zurich, Switzerland. He became a faculty member of the Physics Department at ETH Zurich in April 2001. After his election as Thomas Straumann-Chair for Materials Science in Medicine at the University of Basel, Switzerland and his appointment at the Surgery Department of the University Hospital Basel in September 2006, he founded the Biomaterials Science Center in March 2007. Currently, this center hosts more than 20 researchers dealing with nanotechnology-based artificial muscles for incontinence treatment, smart nanocontainers to treat cardiovascular diseases, high-resolution $\mathrm{X}$-ray imaging to visualize the human body down to the molecular level, computational sciences of tissues in health and disease and other applications of nanosciences in medicine. The mission of the research team can be summarized by using physical principles for human health. Professor Müller teaches physics and materials science at the ETH Zurich and the University of Basel and currently supervises doctoral students from medicine, dentistry, physics, nanosciences and biomedical engineering. He was elected as Fellow of SPIE in 2014 and as an active member of the European Academy of Sciences and Arts in 2015. 